\title{
Correction to: Genetic dissection of grain architecture-related traits in a winter wheat population
}

Matías Schierenbeck ${ }^{1,2,3^{*}}$, Ahmad M. Alqudah ${ }^{4^{*}}$, Ulrike Lohwasser ${ }^{1}$, Rasha A. Tarawneh', María Rosa Simón ${ }^{2,3}$ and Andreas Börner ${ }^{1}$

Correction to: BMC Plant Biol 21, 417 (2021)

https://doi.org/10.1186/s12870-021-03183-3

Following publication of the original article [1], there is an omission in the affiliation of one of the co-authors (María Rosa Simón). The correct affiliation is María Rosa Simón $^{2,3}$.

The original article has been corrected.
Published online: 29 September 2021

\section{Reference}

1. Schierenbeck M, Alqudah AM, Lohwasser U, et al. Genetic dissection of grain architecture-related traits in a winter wheat population. BMC Plant Biol. 2021;21:417 https://doi.org/10.1186/s12870-021-03183-3.

\section{Author details}

${ }^{1}$ Genebank Department, Leibniz Institute of Plant Genetics and Crop Plant Research (IPK OT Gatersleben), Corrensstr 3, D-06466 Seeland, Germany. ${ }^{2}$ Cereals, Faculty of Agricultural Sciences and Forestry, National University of La Plata, La Plata, Argentina. ${ }^{3}$ CONICET CCT La Plata.La Plata, Buenos Aires, Argentina. ${ }^{4}$ Department of Agroecology, Aarhus University at Flakkebjerg, Forsøgsvej 1, 4200 Slagelse, Denmark. 